ANDREA MOURA RODRIGUES ${ }^{1}$

Letícia Maria de Oliveira ${ }^{2}$

KaRINA DE FALCO MARTINS ${ }^{3}$

Carlos Antônio Del Roy ${ }^{3}$

Marair Gracio Ferreira SartorI ${ }^{4}$

Manoel joão Batista Castello Girão 5

Rodrigo de AquinO CASTRO ${ }^{6}$

\title{
Fatores de risco para o prolapso genital em uma população brasileira
}

\author{
Risk factors for genital prolapse in a Brazilian population
}

\section{Artigo original}

\section{Palavras-chave \\ Fatores de risco Prolapso uterino Estudos de caso e controle Brasil/etnologia \\ Keywords \\ Risk factors Uterine prolapsed Case-control studies Brazil/etnology}

\section{Resumo}

OBJETIVO: avaliar os fatores de risco para o desenvolvimento de prolapso genital na população brasileira. MÉTODOS: estudo caso-controle envolvendo 316 pacientes que foram submetidas a estadiamento de prolapso, utilizando-se o sistema de quantificação de prolapso dos órgãos pélvicos. As pacientes foram divididas em dois grupos. No Grupo Caso, foram incluídas 107 pacientes com prolapso nos estádios III ou IV, e no Controle, 209 mulheres com estádios $\mathrm{O}$ ou $\mathrm{l}$. As mulheres selecionadas respondiam à anamnese na qual eram questionadas sobre a presença de possíveis fatores de risco para prolapso genital, tais como: idade, idade da menopausa, paridade, tipos de parto (vaginal, cesariana ou fórcipe), ocorrência de macrossomia fetal, história familiar em parentes de primeiro grau de distopia genital, tosse crônica e constipação intestinal. RESULTADOS: as variáveis que se mostraram diferentes entre os grupos foram: idade, índice de massa corpórea, paridade, número de partos vaginais, de cesarianas, de partos fórcipe, peso do recém-nascido e história familiar positiva para prolapso. Raça, idade da menopausa, tosse crônica e constipação intestinal não se mostraram diferentes entre os grupos. Após a regressão logística, somente três variáveis se apresentaram como fatores de risco independentes: presença de pelo menos um parto vaginal, macrossomia fetal e história familiar positiva. A cesariana se mostrou como fator protetor. CONCLUSÕES: na população brasileira, os fatores de risco independentes para prolapso foram a presença de pelo menos um parto normal, macrossomia fetal e história familiar positiva para distopia.

\section{Abstract}

PURPOSE: to evaluate risk factors for the development of genital prolapse in the Brazilian population. METHODS: case-control study involving 316 patients submitted to prolapse staging, according to the pelvic organ prolapse quantification system. The patients were divided into two groups: in the Case Group there were 107 patients with prolapse at stage III or IV, and in the Control Group, 209 women at stage $\mathrm{O}$ or I. In the anamnesis, the selected women have been questioned about the presence of possible risk factors for genital prolapse, such as: age, menopause age, parturition, delivery type (vaginal, caesarean section or forceps), occurrence of fetal macrosomia, family history of genital dystopia in first degree relatives, chronic cough and intestinal constipation. RESULTS: The variables that were different between the groups were: age, body mass index, parturition, number of vaginal, caesarean section or forceps deliveries, newborn weight and positive family history for prolapse. Race, menopause age, chronic cough and intestinal constipation did not present differences between the groups. After logistic regression, only three variables have been shown to be independent risk factors: presence of at least one vaginal delivery, fetal macrosomia and positive family history for dystopia. Cesarean section was shown to be a protective factor. CONCLUSION: in the Brazilian population, the independent risk factor for genital prolapse were: personal antecedent of at least one vaginal delivery, fetal macrosomia and family history of dystopia.
Correspondência:

Andrea Moura Rodrigues Rua Adolfo Pereira, 233/102 - Anchieto CEP 30310-350 - Belo Horizonte (MG), Brasil Fone: (31) 3221-0868 Fax: (31) 3273-5050 E-mail: deamrodrigues@hotmail.com
Setor de Uroginecologia e Cirurgia Vaginal da Escola Paulista de Medicina da Universidade Federal de São Paulo - UNIFESP - São Paulo (SP), Brasil.

' Uroginecologista pela Escola Paulista de Medicina da Universidade Federal de São Paulo - UNIFESP - São Paulo (SP), Brasil. ${ }^{2}$ Chefe de Ambulatório de Uroginecologia da Escola Paulista de Medicina da Universidade Federal de São Paulo - UNIFESP - São Paulo (SP), Brasil.

${ }^{3}$ Pós-graduandos em Uroginecologia e Cirurgia Vaginal da Escola Paulista de Medicina da Universidade Federal de São Paulo UNIFESP - São Paulo (SP), Brasil.

4 Professora Livre-docente, Chefe da Disciplina de Ginecologia Geral da Escola Paulista de Medicina da Universidade Federal de São Paulo - UNIFESP - São Paulo (SP), Brasil.

5 Professor Titular de Ginecologia da Escola Paulista de Medicina da Universidade Federal de São Paulo - UNIFESP - São Paulo (SP), Brasil.

- Professor Adjunto da Disciplina de Ginecologia da Escola Paulista de Medicina da Universidade Federal de São Paulo - UNIFESP - São Paulo (SP), Brasil. 


\section{Introdução}

O prolapso genital é uma condição ginecológica que não ameaça a vida, mas é causa importante de morbidade. É doença comum que pode afetar intensamente a qualidade de vida das pacientes, causando impacto psicológico, social e financeiro ${ }^{1}$. As informações epidemiológicas desta doença são difíceis de serem obtidas, uma vez que muitas mulheres escondem o problema ou o aceitam como consequência natural do envelhecimento ou dos partos vaginais ${ }^{2}$. A prevalência estimada é de $21,7 \%$ em mulheres de 18 a 83 anos, chegando a $30 \%$ nas pacientes entre 50 e 89 anos $^{3,4}$. Aos 80 anos, $11,1 \%$ das mulheres têm ou tiveram indicação cirúrgica para a correção do prolapso genital ou de incontinência urinária ${ }^{5}$. O prolapso representa um problema socioeconômico importante. A cada ano cerca de 300 a 400 mil mulheres norte-americanas são submetidas a procedimentos cirúrgicos para correção de prolapso pélvico e incontinência urinária, com custo anual de 1 bilhão de dólares ${ }^{6,7}$.

Segundo dados do Instituto Brasileiro de Geografia e Estatística (IBGE), desde 1970, o perfil demográfico brasileiro vem se transformando. Em decorrência da redução da mortalidade proporcionada pelos avanços médicos e das taxas de natalidade ocasionada pela difusão dos métodos contraceptivos, a população brasileira caminha rumo a um padrão demográfico com predominância de adultos e idosos ${ }^{8}$. É estimado que em 2050 o Brasil contará com aproximadamente 9 milhões de mulheres com 80 anos ou mais?

Frente ao envelhecimento da população, à grande morbidade proporcionada pelo prolapso genital e aos elevados custos do tratamento, é possível concluir que há um importante problema de saúde pública. Nos próximos 30 anos é esperado que o número de mulheres que procurarão cuidados médicos por desordens no assoalho pélvico irá dobrar ${ }^{4}$.

O prolapso é considerado uma hérnia do conteúdo pélvico e/ou intraperitoneal no canal vaginal e vários fatores de risco para sua ocorrência são sugeridos. A doença pode ser atribuída a uma combinação de condições, que varia de paciente para paciente ${ }^{10}$. Fatores de risco bem estabelecidos incluem idade, paridade, histerectomia, cirurgias prévias para correção de distopia genital e desordens do colágeno. Porém, o papel de outros fatores potenciais ainda são controversos, como tipo de parto, obesidade, peso do recém-nascido, tosse crônica e história familiar ${ }^{10,11}$.

Com base nesses fatos foi proposta a avaliação dos fatores de risco para o desenvolvimento de prolapso em uma amostra da população brasileira.

\section{Métodos}

Todas as etapas deste trabalho seguiram as normas de boas práticas em estudos clínicos envolvendo seres humanos, de acordo com a resolução 196/96 do Conselho Nacional de Saúde, e foram aprovadas previamente pelo Comitê de Ética em Pesquisa da Escola Paulista de Medicina da Universidade Federal de São Paulo (UNIFESP-EPM).

É um estudo caso-controle no qual foram selecionadas 316 mulheres no Ambulatório de Uroginecologia e Cirurgia Vaginal e no Ambulatório do Climatério, ambos pertencentes ao Departamento de Ginecologia da UNIFESP-EPM, atendidas no período de agosto de 2006 a agosto de 2007. As pacientes assinaram o termo de consentimento livre e esclarecido aprovado pelo Comitê de Ética em Pesquisa da UNIFESP-EPM.

As pacientes foram submetidas a exame ginecológico, no qual era realizado o estadiamento do prolapso genital utilizando o sistema de quantificação de prolapso dos órgãos pélvicos (POP-Q), padronizado pela International Continence Society (ICS $)^{12}$. Segundo este sistema, o prolapso é expresso em centímetros acima (número negativo) ou abaixo do hímen (número positivo). Há seis pontos de referência: dois na parede anterior $(\mathrm{Aa}, \mathrm{Ba})$, dois na parede posterior ( $\mathrm{Ap}, \mathrm{Bp})$, um no colo uterino ou cúpula vaginal $(C)$ e um ponto no fórnice posterior (D). O ponto Aa localiza-se na linha média da parede vaginal anterior, $3 \mathrm{~cm}$ acima do meato uretral externo. Sua posição pode variar de $-3 \mathrm{a}+3 \mathrm{~cm}$. $\mathrm{O}$ ponto $\mathrm{Ba}$ é o ponto de maior procidência da parede vaginal anterior. Os pontos Ap e Bp são análogos aos pontos $\mathrm{Aa}$ e $\mathrm{Ba}$, porém na parede vaginal posterior. Além disso, são medidos o comprimento vaginal total (CVT), o hiato genital, que consiste na medida da uretra ao fórnice vaginal, e o corpo perineal, que é a medida do fórnice ao ânus. Assim, o prolapso genital é classificado em cinco estádios: estádio 0 (ausência de prolapso $\mathrm{Aa}, \mathrm{Ba}, \mathrm{Ap}$ e Bp estão em $-3 \mathrm{~cm}$ e os pontos $\mathrm{C}$ e D estão entre $-2 \mathrm{~cm}$ e o CVT); estádio I (o ponto de maior prolapso está localizado até $1 \mathrm{~cm}$ acima do hímen $(-1 \mathrm{~cm})$ ); estádio II (o ponto de maior prolapso está entre -1 e $+1 \mathrm{~cm}$ ); estádio III (a porção mais distal do prolapso está mais do que $1 \mathrm{~cm}$ abaixo do hímen, porém não atinge o CVT menos $2 \mathrm{~cm}$ ); estádio IV (eversão vaginal completa. A porção mais distal do prolapso é deslocada, no mínimo, o comprimento total da vagina menos $2 \mathrm{~cm}$ (Figura 1$)^{12}$ ).

Após o exame físico, as pacientes foram divididas em dois grupos. O Grupo Controle foi composto por 209 pacientes nos estádios 0 e I (POP-Q/ICS) e o Grupo Caso por 107 mulheres com estádios III e IV (POP-Q/ ICS). Foi medido peso e altura para cálculo do índice de massa corpórea (IMC).

As mulheres selecionadas responderam à anamnese dirigida na qual foram questionadas sobre a presença de possíveis fatores de risco para prolapso genital, tais 
como: idade, idade da menopausa, paridade, tipos de parto (vaginal, cesariana ou fórcipe), ocorrência de macrossomia fetal, história familiar em parentes de primeiro grau de distopia genital, tosse crônica (história de asma ou doença pulmonar obstrutiva crônica) e constipação intestinal desde a infância ou adolescência. A macrossomia fetal foi definida pela história de parto de recém-nascido com peso igual ou maior do que $4.000 \mathrm{~g}^{13}$. Em relação à raça, as mulheres foram classificadas em brancas ou não-brancas. As pacientes não-brancas eram as consideradas mulatas, negras e amarelas. Esta classificação foi utilizada devido à intensa miscigenação presente na população brasileira, que impede a diferenciação precisa de grupos étnicos apenas pela característica física ${ }^{14}$.

Todas as pacientes tinham história clínica compatível com pós-menopausa (ausência de fluxo menstrual há pelo menos um ano) e não usavam terapia hormonal. Não foram incluídas no estudo pacientes que já haviam sido submetidas a cirurgias vaginais, que apresentavam prolapso genital no estádio II (ICS) e que não desejavam participar do estudo.

As análises estatísticas foram realizadas utilizando o pacote estatístico Statistical Package for Social Sciences, v. 14.0 (SPSS). Para comparação das variáveis qualitativas (raça, história familiar, tosse crônica e constipação intestinal) foi utilizado o teste do $\chi^{2}$. Em relação aos dados quantitativos (idade, IMC, idade da menopausa, paridade, número de partos vaginais, cesarianas e fórcipe, peso do recém-nascido) foi utilizado, inicialmente, o teste Kolmogorov-Smirnov para verificar se os dados a serem analisados estavam distribuídos de acordo com os padrões de normalidade. Para verificar a associação entre os grupos estudados e os dados quantitativos, foi utilizado o teste de Mann-Whitney U, exceto para a variável IMC, em que se utilizou o teste $t$, por ser esta a única variável com distribuição normal. $\mathrm{O}$ valor de significância estatística foi estabelecido em 5\%, ou $\mathrm{p}<0,05$. O risco para a presença de prolapso dos órgãos pélvicos foi estimado por meio do cálculo do valor de Odds Ratio (OR) e intervalos de confiança (IC), utilizando a regressão logística binária.

\section{Resultados}

Os grupos foram estatisticamente heterogêneos considerando as variáveis clínicas e demográficas, exceto pela raça, idade média da menopausa, presença de tosse crônica e constipação intestinal (Tabela 1).

Os fatores de risco independentes para o desenvolvimento de prolapso foram determinados por meio do cálculo do OR pelo modelo de regressão logística. O fato de ter tido pelo menos um parto vaginal aumenta

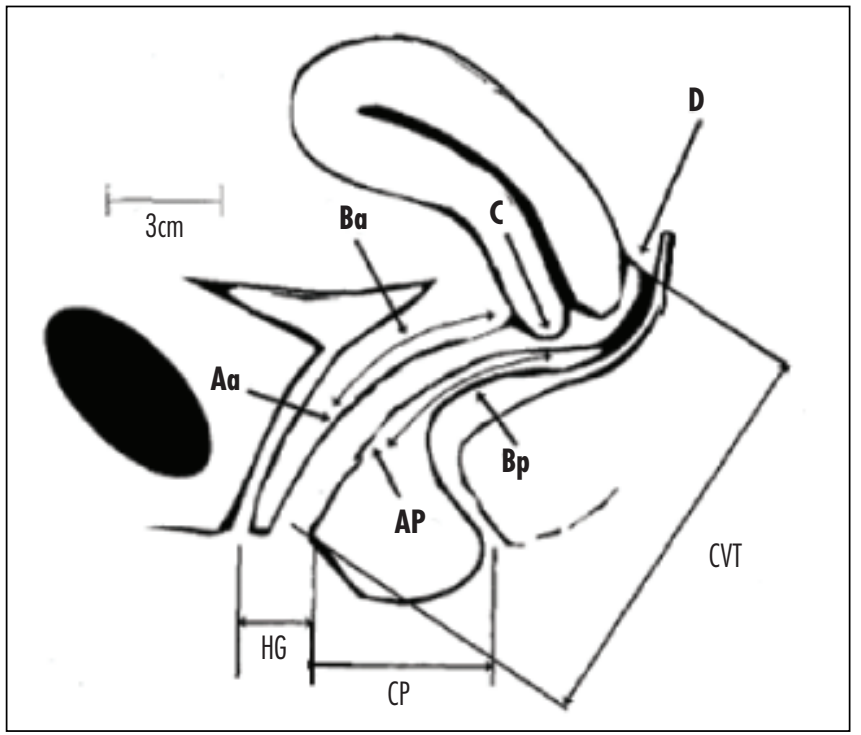

Figura 1 - Pontos de referência para estadiamento do prolapso genital: Aa, $\mathrm{Ba}, \mathrm{Ap}, \mathrm{Bp}, \mathrm{C}, \mathrm{D}$, hiato genital (HG), corpo perineal (CP) e comprimento vaginal total $(C V T)^{12}$.

Tabela 1 - Características clínicas e demográficas dos Grupos Controle (prolapsos nos estádios 0 e I) e Caso (prolapso nos estádios III e IV)

\begin{tabular}{lccc}
\hline Variável & $\begin{array}{c}\text { Grupo Controle } \\
(\mathbf{n}=209)\end{array}$ & $\begin{array}{c}\text { Grupo Caso } \\
(\mathbf{n}=107)\end{array}$ & Valor de p \\
\hline Média de idade (anos) & 60,8 & 66,3 & $<0,0001$ \\
IMC médio (kg/m²) & 26,5 & 27,8 & 0,02 \\
\hline Raça & & & \\
$\quad$ Branca & $58,4 \%$ & $63,6 \%$ & \\
$\quad$ Não-branca & $41,6 \%$ & $36,4 \%$ & 0,37 \\
Paridade (média) & 2,01 & 4,5 & $<0,0001$ \\
Partos vaginais (média) & 1,03 & 4,01 & $<0,0001$ \\
Cesarianas (média) & 0,86 & 0,24 & $<0,0001$ \\
Partos fórcipe (média) & 0,13 & 0,25 & 0,01 \\
Peso do recém-nascido & 3.451 & 3.820 & 0,0002 \\
(média) & & & \\
Idadade da menopausa & 47,7 & 48,8 & 0,15 \\
(média) & & & \\
História familiar positivava & $17,2 \%$ & $28 \%$ & 0,02 \\
para prolapso & & & \\
Tosse crônica & $10,5 \%$ & $11,2 \%$ & 0,85 \\
Constipação infestinal & $31,6 \%$ & $27,1 \%$ & 0,41 \\
\hline
\end{tabular}

IMC=índice de massa corpórea.

o risco de prolapso em aproximadamente sete vezes $(\mathrm{OR}=7,22$; IC95\%=1,84-28,27). Ter se submetido ao parto de recém-nascido macrossômico aumenta o risco em cerca de três vezes $(\mathrm{OR}=2,9$; IC95\%=1,24-6,79). A cesariana se mostrou como fator de proteção $(\mathrm{OR}=0,43$; IC95\% $=0,24-0,78)$. A presença de história familiar positiva para prolapso aumenta o risco em 2,3 vezes $(\mathrm{OR}=2,27$; IC95\% $1,04-4.9)$. Apesar de serem diferentes entre os dois grupos, idade $(\mathrm{OR}=1,05$; IC95\%=1,0-1,1), IMC $(\mathrm{OR}=1,08 ; \mathrm{IC} 95 \%=1,0-1,1)$ e parto fórcipe $(\mathrm{OR}=1,0$; IC95\%=0,59-1,91) não se revelaram como fatores de risco independentes (Tabela 2). 
Tabela 2 - Fatores de risco independentes para prolapso genital

\begin{tabular}{l|c|c}
\hline \multicolumn{1}{l}{ Variáiveis } & Valor de $\mathrm{p}$ & OR (IC95\%) \\
\hline $\begin{array}{l}\text { Presença de pelo menos um parto } \\
\text { normal }\end{array}$ & 0,005 & $7,22(1,84-28,27)$ \\
\hline Idade & 0,02 & $1,05(1,0-1,1)$ \\
IMC & 0,025 & $1,08(1,0-1,1)$ \\
Partos fórcipe & 0,82 & $1,0(0,59-1,91)$ \\
Cesarianas & 0,006 & $0,43(0,24-0,78)$ \\
História familiar positiva & 0,038 & $2,27(1,04-4,9)$ \\
Peso do RN $\geq 4.000 \mathrm{~g}$ & 0,014 & $2,9(1,24-6,79)$ \\
\hline
\end{tabular}

$\mathrm{OR}=$ Odds Ratio; $I C=$ intervalo de confiança; $I M C=$ índice de massa corpórea; $\mathrm{RN}=$ recém-nascido.

\section{Discussão}

Os resultados obtidos no presente estudo estão de acordo com a maioria dos trabalhos publicados da literatura mundial. As variáveis: idade, IMC, paridade, número de partos normais, número de cesarianas, número de partos fórcipe, peso do recém-nascido e história familiar positiva para prolapso foram significativamente diferentes entre os dois grupos estudados. Porém, apenas três delas se apresentaram como fatores de risco independentes para prolapso genital nesta amostra: a presença de pelo menos um parto normal, macrossomia fetal e história familiar positiva para distopia. O parto cesariano se mostrou como fator protetor.

O prolapso parece ser uma condição multifatorial. Vários estudos identificaram fatores de risco para esta condição, tais como parto vaginal, hipoestrogenismo, macrossomia fetal, uso de fórcipe e vácuo-extrator, episiotomia, histerectomia e cirurgias prévias para correção de distopia genital ${ }^{12,15,16}$.

O parto vaginal se destaca como o principal fator de risco ${ }^{6}$. Quando a apresentação fetal passa pela pelve, a compressão, distensão e o estiramento podem lesar o nervo pudendo, o suporte fascial e o músculo levantador do ânus. Este músculo, juntamente com a fáscia endopélvica, são os responsáveis por manter as vísceras pélvicas na posição anatômica correta ${ }^{17}$. Em estudos epidemiológicos, a distopia genital está fortemente associada à paridade e principalmente ao parto normal e ao peso de nascimento do recém-nascido ${ }^{15,16,18}$. Swift, Pound e Dias ${ }^{19}$ correlacionaram, por meio de estudos de regressão logística, o número de gestações, número de partos vaginais e o maior peso dos filhos recémnascidos. A variável que mostrou associação mais forte com prolapso foi o peso do recém-nascido. Na nossa análise, o principal fator de risco foi o parto vaginal, seguido pela macrossomia fetal.

Entretanto, o parto vaginal isoladamente não explica a ocorrência de prolapso, pois este também está presente em mulheres nulíparas ${ }^{15,18}$ e está ausente na maioria das mulheres que foram submetidas a parto vaginal, ocorrendo geralmente várias décadas após o trauma do parto ${ }^{20}$. Há indícios de que fatores genéticos associados a fatores ambientais participem da gênese desta doença. No presente estudo, a presença de história familiar positiva aumentou em 2,3 vezes a chance de se desenvolver prolapso. McLennan et al. ${ }^{10}$ encontraram um aumento do risco em 1,4 vezes nas pacientes que tinham história familiar de prolapso e/ou hérnia. A possibilidade de transmissão familiar do prolapso genital foi verificada num estudo que incluiu 182 pacientes nos estádios III e IV. Foi encontrado um aumento de cinco vezes para o diagnóstico de prolapso nas mães, irmãs e avós das pacientes. Dentro destas famílias, a transmissão da doença seguiu padrão de herança dominante com alta penetrância ${ }^{21}$. Em outro estudo, foram encontradas diferenças significativas na expressão gênica de proteínas estruturais relacionadas à actina, miosina $\mathrm{e}$ matriz extracelular no músculo pubococcígeo de pacientes com prolapsos avançados quando comparadas a pacientes sem prolapso ${ }^{20}$.

Doenças crônicas que resultam em aumento da pressão intra-abdominal parecem estar envolvidas na etiologia do prolapso ${ }^{22,23}$. Tosse crônica, obesidade e constipação são as condições mais relatadas. Mas há poucos dados na literatura que confirmam tal fato. Nesta análise, tosse crônica e constipação intestinal não foram variáveis com distribuição diferente entre os grupos. A obesidade se mostrou associada ao desenvolvimento de prolapso somente quando acompanhada de outros possíveis fatores de risco. $\mathrm{Na}$ análise de regressão logística, IMC > 25 não foi fator de risco independente.

Com os resultados obtidos neste estudo, fica claro que o prolapso genital é realmente uma condição multifatorial. Para o seu desenvolvimento, contribuem fatores sobre os quais não é possível intervir, como idade e história familiar, e outros que podem ser alterados, como elevado IMC, paridade, tipos de parto e macrossomia fetal. Os ginecologistas e obstetras são os médicos responsáveis por cuidar de mulheres com prolapso e, também, os que têm as melhores oportunidades para atuar na sua prevenção. O parto é uma das oportunidades ideais para isso, pois o parto vaginal é o principal fator de risco e ocorre sob a supervisão do médico. Cabe ao obstetra indicar a via adequada de parto, reconhecer possíveis lesões no assoalho pélvico provocadas pelo parto e fornecer tratamento adequado em tempo hábil.

Até onde se conhece, este é o primeiro trabalho que determina fatores de risco para prolapso, com grande número de pacientes na população brasileira. Mais estudos precisam ser realizados, principalmente no campo da biologia molecular, para determinar outros fatores relevantes associados ao prolapso genital e traçar estratégias eficazes para a profilaxia da doença. 
1. Jelovsek JE, Barber MD. Women seeking treatment for advanced pelvic organ prolapse have decreased body image and quality of life. Am J Obstet Gynecol. 2006; 194(5): 1455-61.

2. Bidmead J, Cardozo LD. Pelvic floor changes in the older woman. Br J Urol. 1998;82 Suppl 1:18-25.

3. Swift $S$, Woodman $P, O^{\prime}$ Boyle A, Kahn M, Valley M, Bland D, et al. Pelvic Organ Support Study (POSST): the distribution, clinical definition, and epidemiologic condition of pelvic organ support defects. Am J Obstet Gynecol. 2005;192(3):795-806.

4. Luber KM, Boero S, Choe JY. The demographics of pelvic floor disorders: current observations and future projections. Am J Obstet Gynecol. 2001;184(7):1496-501.

5. Olsen AL, Smith VJ, Bergstrom JO, Colling JC, Clark AL. Epidemiology of surgically managed pelvic organ prolapse and urinary incontinence. Obstet Gynecol. 1997;89(4):501-6.

6. Delancey JO. The hidden epidemic of pelvic floor dysfunction: achievable goals for improved prevention and treatment. Am J Obstet Gynecol. 2005;192(5): 1488-95.

7. Subak LL, Waetjen E, van den Eeden S, Thom DH, Vittinghoff E, Brown JS. Cost of pelvic organ prolapse surgery in the United States. Obstet Gynecol. $2001 ; 98(4): 646-51$.

8. Instituto Brasileiro de Geografia e Estatística (IBGE). Fundo de População das Nações Unidas (UNFPA). Indicadores sociodemográficos prospectivos para ○ Brasil 1991-2030 [documento da Internet]. Rio de Janeiro: IBGE/UNFPA; out. 2006 [citado 5 fev 2008]. Disponível em: http://www.ibge. gov.br/home/estatistica/populacao/projecao_da_populacao/ publicacao_UNFPA.pdf

9. Oliveira JC, Albuquerque FRPC, Lins IB. Projeção da população do Brasil por sexo e idade para o período de 1980-2050: revisão de 2004. Metodologia e resultados. Estimativas anuais e mensais da população do Brasil e das unidades da federação: 1980-2020. Metodologia. Estimativa das populações municipais. Metodologia [Internet]. Rio de Janeiro: IBGE; out. 2004 [citado 2008 Fev 5]. Disponível em: http://www.ibge.gov.br/home/ estatistica/populacao/estimativa2004/ metodologia.pdf

10. McLennan MT, Harris JK, Kariuki B, Meyer S. Family history as a risk factor for pelvic organ prolapse. Int Urogynecol J Pelvic Floor Dysfunct. 2008;19(8):1063-9.
11. Kim CM, Jeon M, Chung DJ, Kim SK, Kim JW, Bai SW. Risk factors for pelvic organ prolapse. Int J Gynaecol Obstet. 2007;98(3):24851.

12. Bump RC, Mattiasson A, Bo K, Brubaker LP, DeLancey JO, Klarskov $P$, et al. The standardization of terminology of female pelvic organ prolapse and pelvic floor dysfunction. Am J Obstet Gynecol. 1996;175(1):10-7.

13. Pates JA, Mclntire DD, Casey BM, Leveno KJ. Predicting macrosomia. J Ultrasound Med. 2008;27(1):39-43.

14. Parra FC, Amado RC, Lambertucci JR, Rocha J, Antunes CM, Pena SD. Color and genomic ancestry in Brazilians. Proc Natl Acad Sci USA. 2003;100(1):177-82.

15. Mant J, Painter R, Vessey M. Epidemiology of genital prolapse: observations from the Oxford Family Planning Association Study. Br J Obstet Gynaecol. 1997; 104(5):579-85.

16. Swift SE. The distribution of pelvic organ support in a population of female subjects seen for routine gynecologic health care. Am J Obstet Gynecol. 2000;1 83(2):277-85.

17. Tegerstedt G, Miedel A, Maehle-Schmidt M, Nyrén O, Hammarström M. Obstetric risk factors for symptomatic prolapse: a populationbased approach. Am J Obstet Gynecol. 2006;194(1):75-81.

18. Dietz HP, Eldridge A, Grace $M$, Clarke B. Pelvic organ descent in young nulligravid women. Am J Obstet Gynecol. 2004;191(1):95-9.

19. Swift SE, Pound T, Dias JK. Case-control study of etiologic factors in the development of severe pelvic organ prolapse. Int Urogynecol J Pelvic Floor Dysfunct. 2001;12(3): 187-92.

20. Visco AG, Yuan L. Differential gene expression in pubococcygeus muscle from patients with pelvic organ prolapse. Am J Obstet Gynecol. 2003;189(1):102-12.

21. Jack GS, Nikolova G, Vilain E, Raz S, Rodríguez LV. Familial transmission of genitovaginal prolapse. Int Urogynecol J Pelvic Floor Dysfunct. 2006;17(5):498-501.

22. Bump RC, Norton PA. Epidemiology and natural history of pelvic floor dysfunction. Obstet Gynecol Clin North Am. 1998;25(4):723-46.

23. Bodner-Adler B, Shrivastava C, Bodner K. Risk factors for uterine prolapse in Nepal. Int Urogynecol J Pelvic Floor Dysfunct. 2007; 18(11):1343-6. 